\title{
A Negative Imaginary Approach to Modeling and Control of a Collocated Structure
}

\author{
Bharath Bhikkaji, S. O. Reza Moheimani, Fellow, IEEE, and Ian R. Petersen, Fellow, IEEE
}

\begin{abstract}
A transfer-function is said to be negative imaginary if the corresponding frequency response function has a negative definite imaginary part (on the positively increasing imaginary axis). Negative imaginary transfer-functions can be stabilized using negative imaginary feedback controllers. Flexible structures with compatible collocated sensor/actuator pairs have transferfunctions that are negative imaginary. In this paper a model structure that typically represents a collocated structure is considered. An identification algorithm which enforces the negative imaginary constraint is proposed for estimating the model parameters. A feedback control technique, known as integral resonant control (IRC), is proposed for damping vibrations in collocated flexible structures. Conditions for the stability of the proposed controller are derived, and shown that the set of stabilizing IRCs is convex. Finally, a flexible beam with two pairs of collocated piezoelectric actuators/sensors is considered. The proposed identification scheme is used determining the transfer-function and an IRC is designed for damping the vibrations. The experimental results obtained are reported.
\end{abstract}

Index Terms-Integral resonant control, linear feedback control, negative imaginary systems, piezoelectric actuators.

\section{INTRODUCTION}

$\mathbf{F}$ LEXIBLE structures are highly resonant systems that are susceptible to high amplitude oscillations even in presence of mild disturbances. Several industrial and scientific devices include components that can be classified as flexible structures, [1]-[9] and [10]. High amplitude oscillations of these components can result in significant loss of precision and possible breakdown if the amplitude crosses the elastic limit. Thus, there is a need to damp or control the oscillations that arise in flexible structures. Here, as in most control literature, [1]-[7], [11] and [10] flexible structures under consideration are restricted to linear systems that are controllable and observable and have complex conjugate poles with small real parts.

Designing controllers to damp vibrations in flexible structures have always been pursued with a lot interest. Recently, there has

Manuscript received August 4, 2010; revised November 1, 2010; accepted January 22, 2011. Date of publication April 19, 2011; date of current version May 4, 2012. Recommended by Technical Editor N. Chaillet.

B. Bhikkaji is with the Department of Electrical Engineering, Indian Institute of Technology Madras, Chennai, 600036, India (e-mail: Bharath.Bhikkaji@ iitm.ac.in).

S. O. R. Mohiemani is with the School of Electrical Engineering and Computer Science, University of Newcastle, Callaghan, NSW, 2308, Australia (e-mail: Reza.Moheimani@newcastle.edu.au).

I. R. Petersen is with the School of Information Technology and Electrical Engineering, University of New South Wales, Australian Defence Force Academy Campus, Canberra, Australia (e-mail: irp@ee.adfa.edu.au).

Color versions of one or more of the figures in this paper are available online at http://ieeexplore.ieee.org.

Digital Object Identifier 10.1109/TMECH.2011.2123909 been a significant raise in focus on control design for flexible structures with collocated sensors and actuators, [12]-[17]. Collocated structures are known to offer robust stability properties. However, clear mathematical characterizations of such properties have not been provided. This paper deals with identification and vibration control of flexible structures with collocated sensors and actuators. Here, a novel identification scheme is proposed for modeling these systems, and a new control design scheme known as integral resonant control (IRC) is presented to augment their damping.

In general, dynamics of flexible structures are characterized by partial differential equations, [1], [2], and [10]. Therefore, they posses an infinite number of lightly damped resonant modes. Alternatively, the transfer-functions obtained from the PDEs are of infinite order with poles very close to the imaginary axis. However, in practice, the PDE models are discretized to form finite order models. Hence, the infinite order transfer functions are truncated and only the first few modes are retained, [10] and [18]. Using the truncated model, feedback controllers are designed to damp the resonant modes of the structure. The discarded modes, referred to as the out-of-bandwidth modes or the residual modes, often play a limited part in the controller design. The presence of these uncontrolled truncated modes often lead to what is known as the spill-over effect, [19], [20], and [18]. That is, the control energy is inadvertently supplied to the residual modes, which may destabilize the closed-loop system.

Under certain conditions, flexible structures with collocated sensors and actuators can be stabilized in the presence of out of bandwidth modes. Velocity feedback, [6] and [10], and resonant controllers [21], are two well known examples of controllers that guarantee unconditional closed-loop stability when implemented on collocated structures. Positive position feedback (PPF), [22], [23], and [24], is another technique which is not sensitive to the spill-over effect, and has similar stability properties. Unlike velocity feedback and resonant control, the frequency response of a PPF controller rolls off at high frequencies, thus leaving the unmodeled high-frequency modes of the plant undisturbed.

Flexible structures with collocated and compatible sensors and actuators lead to negative imaginary transfer functions. State space characterizations of negative imaginary transfer functions were studied in [25], where it was shown that the state space matrices corresponding to a negative imaginary transfer function were constrained by certain linear matrix inequalities (LMIs). In this paper, these LMI constraints are used to develop a system identification framework for negative imaginary systems in general, and for flexible systems with collocated sensors and actuators, in particular. 
In [26], the authors have proposed a feedback control scheme known as the IRC. This technique was developed for a flexible structure with a single collocated sensor/actuator pair, i.e., for the SISO case. It was reported in [26] that IRC achieved remarkable damping. This was validated in the context of atomic force microscopes in [27]. This paper generalizes the work of [26] to the case of flexible structures with multiple collocated sensor/actuator pairs; i.e., to collocated MIMO systems. In particular a mathematical framework for IRC is provided. Stability conditions for IRC are derived and shown that the set of stabilizing controllers is convex. The possibility of making them unconditionally stable is also discussed. Moreover, due to its parametric structure, the IRC rolls off quickly at high frequencies.

It must be mentioned, here, that not all systems exhibit negative imaginary property. Indeed, collocation is an exception than a rule in many cases, [28] and [29]. However, IRC has been successfully applied to approximately collocated systems, such as pizeelectric tubes, [27] and [30].

This paper is formatted as follows: In Section II, the notion of a negative imaginary system is introduced and certain characterizations and properties of negative imaginary systems are reviewed. The subspace-based identification scheme is also presented in Section II. In Section III, the IRC is presented, and the motivations behind this control design scheme are discussed. In Section IV, the IRC scheme is extended to multivariable systems, and the set of stabilizing IRC controllers form a convex set is also shown. An experimental set up with collocated sensor/actuator pairs is considered in Section V, and the identification algorithm presented in Section II is used to estimate a model. This model is then used to design an IRC that is successfully implemented on the structure.

\section{IDENTIFICATION OF NEGATIVE IMAGINARY SYSTEMS}

Systems with collocated sensors and actuators have the same number of inputs and outputs. Hence, a square system with $n$ inputs and $n$ outputs are considered. Let $G(s)$ denote the transfer-function of a square system with $n$ inputs and $n$ outputs with a minimal state space realization

$$
G(s)=\left[\begin{array}{ll}
A & B \\
C & D
\end{array}\right]
$$

Definition: $G(s)$ is said to be negative imaginary if it is Hurwitz and belongs to the set

$$
\mathcal{C} \triangleq\left\{R(s) \in \mathcal{R} H_{\infty}^{n \times n}: \mathrm{j}\left[R(\mathrm{j} \omega)-R^{*}(\mathrm{j} \omega)\right] \geq 0,\right\}
$$

where $\omega \in(0, \infty)$ and $\mathcal{R} H_{\infty}$ denote the set of all real rational and stable $n \times n$ transfer-function matrices.

The following lemma, presented in [25], gives a condition on the structure of $G(s)$ for it to belong to the set $\mathcal{C}$ defined in (1):

Lemma 1: Let $\left[\begin{array}{ll}A & B \\ C & D\end{array}\right]$ be a minimal state space realization of a transfer-function matrix $G(s)$. Then $G(s) \in \mathcal{C}$ if and only if $A$ is Hurwitz, $D$ is symmetric, and there exists an $n \times n$ symmetric matrix $P$, such that

$$
\begin{aligned}
P & >0 \\
A P+P A^{\top} & \leq 0
\end{aligned}
$$

and

$$
B=-A P C^{\top}
$$

Proof: Refer to Lemma 1 in [25].

Flexible structures with collocated actuator/sensor pairs are known to have negative imaginary transfer function matrices. To design a controller for such systems, an efficient method for identifying the transfer functions is needed. In the following a system identification algorithm, based on the subspace frequency domain identification approach, that estimates a negative imaginary transfer function is presented.

Subspace identification of a linear system $G(s)=D+$ $C(s I-A)^{-1} B$ consists of two steps [31]:

1) An estimate of the matrices $A$ and $C$ is calculated using the subspace based approach.

2) Estimates of $B$ and $D$ are obtained by minimizing a least squares criterion.

Details on estimating $A$ and $C$ from given frequency domain data $\left\{G\left(\mathrm{j} \omega_{k}\right)\right\}_{k=0}^{N-1}$ can be found in [31], [32], and [33]. In this paper, only the second step is modified using Lemma 1 to enforce the identification of a negative imaginary system. The first step of our algorithm is identical to that described in [31], and is therefore, not discussed here in the interest of brevity.

Assume that both $A$ and $C$ matrices have been estimated and $\tilde{A}$ and $\tilde{C}$ be the corresponding estimates. Let

$$
E_{k} \triangleq G\left(\mathrm{j} \omega_{k}\right)-\left(D+\tilde{C}\left(\mathrm{j} \omega_{k}-\tilde{A}\right)^{-1} B\right) .
$$

As the system under consideration is known a priori, to be negative imaginary, matrices $B$ and $D$ can be obtained from a solution to the constrained least squares problem

$$
\min _{B, D, P} \sum_{k=0}^{N-1}\left\|E_{k}\right\|^{2}
$$

subject to constraints (2)-(4). Using (4) in (6), the optimization problem can be restated as

$$
\min _{D, P} \sum_{k=0}^{N-1}\left\|G\left(\mathrm{j} \omega_{k}\right)-\left(D-\tilde{C}\left(\mathrm{j} \omega_{k}-\tilde{A}\right)^{-1} \tilde{A} P C^{\top}\right)\right\|^{2}
$$

subject to

$$
\begin{aligned}
& P>0 \\
& \tilde{A} P+P \tilde{A}^{\top} \leq 0
\end{aligned}
$$

and $D$ being symmetric, i.e., $D-D^{\top}=0$. In the standard subspace method estimation of the matrices $D$ and $B$ would involve minimizing the sum of squares of $E_{k},(5)$, without the constraints (2)-(4).

Note that the cost function (7) is quadratic in $P$ and $D$, and the constraints are affine in $P$ and $D$. Hence, the constrained optimization problem is convex. Good numerical solutions to this constrained optimization problem can be obtained using the Matlab package SeDuMi [34]. 


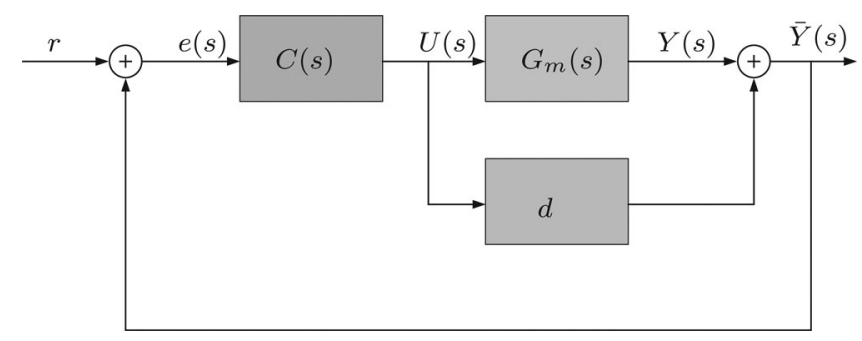

Fig. 1. Closed-loop system with the Integral controller $C(s)=\frac{\Gamma}{s}$ around $G_{m}(s)+d$.

\section{INTEGRAL RESONANT CONTROL OF SISO COLLOCATED SYSTEMS}

Here, for the sake of completeness, the IRC presented in [26] for an SISO collocated flexible system is thoroughly reviewed and presented.

In [26], the authors considered a collocated system represented by a scalar transfer function of the form

$$
G_{m}(s)=\sum_{k=1}^{m} \frac{\psi_{k}}{s^{2}+2 \sigma_{k} \omega_{k} s+\omega_{k}^{2}}+d_{u}
$$

where the quantities $d_{u}, \psi_{k}, \sigma_{k}$, and $\omega_{k}$ are all positive. As (10) denotes a lightly damped flexible structure, the term $2 \sigma_{k} \omega_{k}$ is negligible when compared with $\omega_{k}^{2}$. Significant damping was achieved by adding a feed through term $d$ and wrapping an integral controller $C(s)=\frac{\gamma}{s}$ around the modified plant. The IRC structure is depicted in Fig. 1. In [26], to analyze the stability of the closed-loop system, $G_{m}(s),(10)$, was approximated by

$$
\hat{G}_{m}(s)=\sum_{k=1}^{m} \frac{\psi_{k}}{s^{2}+\omega_{k}^{2}}+d_{u} .
$$

It was shown in [26] that $\hat{G}_{m}(s),(11)$, is equivalent to

$$
\hat{G}_{m}(s)=K \frac{\left(s^{2}+\omega_{z_{1}}^{2}\right) \ldots\left(s^{2}+\omega_{z_{n-1}}^{2}\right)}{\left(s^{2}+\omega_{1}^{2}\right)\left(s^{2}+\omega_{2}^{2}\right) \ldots\left(s^{2}+\omega_{n}^{2}\right)}
$$

where

$$
0<\omega_{1}<\omega_{z_{1}}<\omega_{2}<\omega_{z_{2}} \ldots<\omega_{z_{n-1}}<\omega_{n}
$$

and $K>0$. An implication of the above is that the poles and zeros of $\hat{G}_{m}(s),(11)$, are purely imaginary and their pole-zero map (PZ map) has a pole-zero alternating pattern, as illustrated in Fig. 2(a). By adding a suitable $d$ term to $\hat{G}_{m}(s)$, the zeros can be shifted in such a way that $\bar{G}(s) \triangleq \hat{G}_{m}(s)+d$ has a PZ map with a zero-pole alternating pattern, as illustrated in Fig. 2(b). Alternatively stated, it can be shown that (see [26])

$$
\begin{aligned}
\bar{G}(s) & \triangleq \sum_{k=1}^{n} \frac{\alpha_{k}}{s^{2}+\omega_{k}^{2}}+d \\
& =K^{\prime} \frac{\left(s^{2}+\omega_{z_{1}^{*}}^{2}\right) \ldots\left(s^{2}+\omega_{z_{n}^{*}}^{2}\right)}{\left(s^{2}+\omega_{1}^{2}\right)\left(s^{2}+\omega_{2}^{2}\right) \ldots\left(s^{2}+\omega_{n}^{2}\right)}
\end{aligned}
$$

for all $d<d^{*}<0$, where

$$
0<\omega_{z_{1}^{*}}<\omega_{1}<\omega_{z_{2}^{*}}<\cdots<\omega_{n-1}<\omega_{z_{n}^{*}}<\omega_{n}
$$

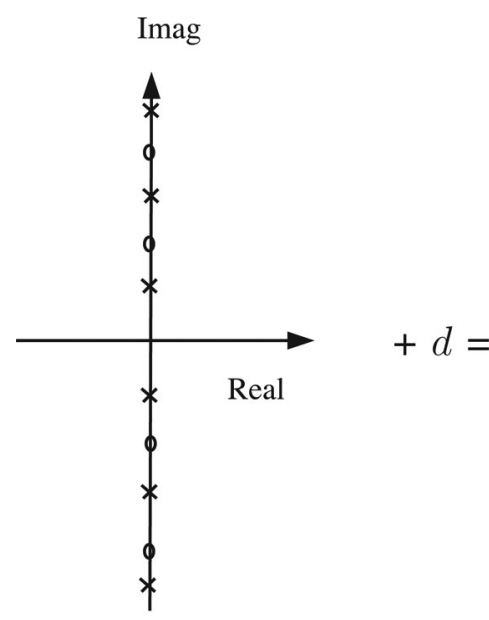

(a)

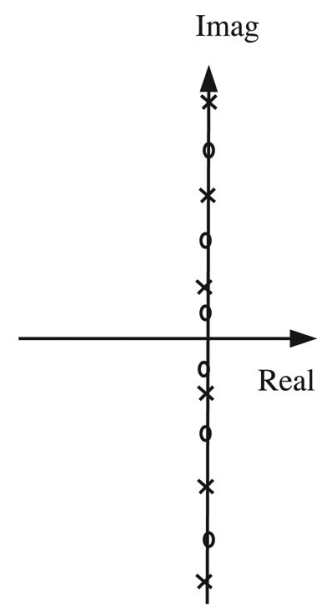

(b)
Fig. 2. Illustration of the Pole-Zero positions of $\hat{G}_{m}(s),(11)$, and $\bar{G}=$ $\hat{G}_{m}(s)+d$, "×" denoting the poles and "o" denoting the zeros.

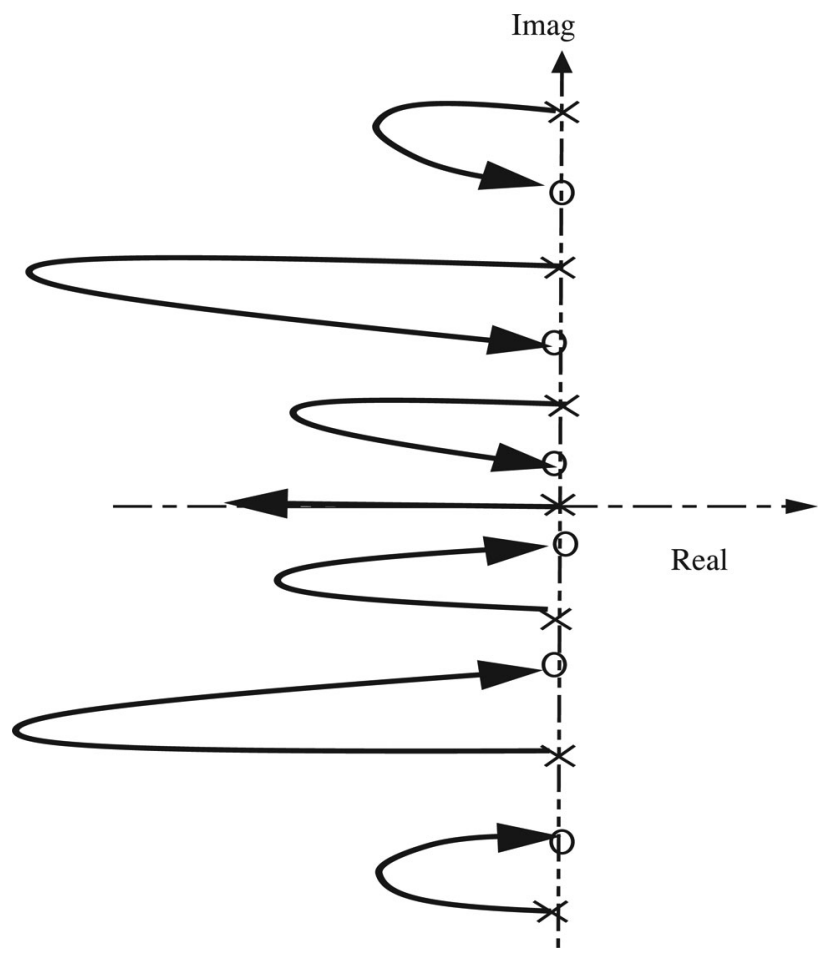

Fig. 3. Illustration of the root locus of $\frac{1}{s} \bar{G}_{m}(s)$.

and

$$
d^{*}=-\left(\sum_{k=1}^{m} \frac{\psi_{k}}{\omega_{k}^{2}}+d_{u}\right)
$$

Due to the zero-pole interlacing in (15), it was observed in [26] that the root-locus plot of $\frac{1}{s} \bar{G}_{m}(s)$ would lie entirely in the left-half plane. Thus, by choosing a reasonable $\gamma$ one could achieve significant damping, see Fig. 3. Note that this amounts to wrapping $C(s)=\frac{\gamma}{s}$ around $\hat{G}_{m}(s)+d$. Therefore, to design an IRC for an SISO collocated system consists of two steps: First, a feed through term has to be added to the plant to place 
a zero between the first pole and the origin; then, an integrator needs to be incorporated, as illustrated in Fig. 1.

However, in [26], no specific reasons were given as to why the root-locus was lying completely in the left-half plane. Though, it was a direct implication of the Hermite-Biehler Theorem (HB Theorem) (see p. 41 of [35]). Furthermore, the approximation made in (11) does note make this a stability proof, but definitely a plausibility argument.

\section{INTEGRAL RESONANT CONTROL OF MIMO COLlOCATED SYSTEMS}

Due to the spatially distributed nature of flexible structures, it may not be possible to control all the required modes using a single actuator/sensor pair. Some of the in-bandwidth modes may be unobservable, uncontrollable or to damp them may require a significant control effort. This leads to the necessity of using multiple actuators and sensors in flexible structures. In this section, the above mentioned IRC scheme is extended to allow for a MIMO system. In particular, we will describe a MIMO IRC structure that covers the SISO controller as a special case. The mathematical machinery used to establish stability of the SISO system cannot be applied to the MIMO case. Our stability proof will utilize the negative imaginary nature of the plant. Furthermore, stability proof does not require approximations of the form (11).

Note from (10) that

$$
\begin{aligned}
G_{m}(\mathrm{j} \omega) & =\sum_{k=1}^{m} \frac{\psi_{k}}{-\omega^{2}+2 \mathrm{j} \sigma_{k} \omega_{k} \omega+\omega_{k}^{2}}+d_{u} \\
& =\sum_{k=1}^{m} \frac{\psi_{k}}{r_{k}}\left(\omega_{k}^{2}-\omega^{2}-2 \mathrm{j} \sigma_{k} \omega_{k} \omega\right)+d_{u}
\end{aligned}
$$

where

$$
r_{k}=\left(\omega_{k}^{2}-\omega^{2}\right)^{2}+\left(2 \sigma_{k} \omega_{k}\right)^{2} .
$$

It is evident from (17) that $G_{m}(\mathrm{j} \omega)$ satisfies the negative imaginary property and, hence, belongs to the set $\mathcal{C}$ defined in (1), for the SISO case, i.e., for $n=1$. A natural extension of the IRC to the MIMO case is to replace the scalar feed-through term $d$ and the scalar gain $\gamma$ by symmetric $n \times n$ matrices $D_{f}$ and $\Gamma$, respectively. Before proving the closed-loop stability, for technical ease, the above mentioned control scheme is rewritten into an equivalent form. This equivalent form makes it easier to analyze the stability in the MIMO case. by

Note, from Fig. 1, that the input to the controller $C(s)$ is given

$$
e(s)=r(s)+\tilde{Y}(s)
$$

where (in the MIMO case)

$$
\tilde{Y}(s)=Y(s)+D_{f} U(s)
$$

with $U(s)$ and $Y(s)$ being the plant inputs and outputs, respectively. The output of the controller, which is the plant input $U(s)$, is given by $U(s)=\frac{\Gamma}{s} e(s)$. Therefore,

$$
U(s)=\frac{\Gamma}{s} r(s)+\frac{\Gamma}{s} Y(s)+\frac{\Gamma}{s} D_{f} U(s)
$$

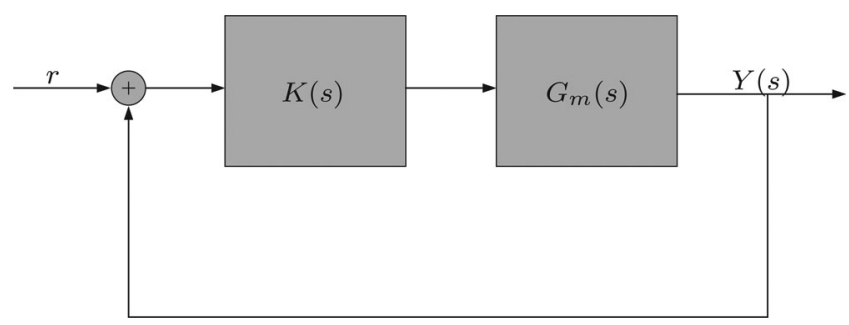

Fig. 4. Closed-loop system with the controller $K(s)$ around $G_{m}(s)$.

which implies

$$
\left(s I-\Gamma D_{f}\right) U(s)=\Gamma r(s)+\Gamma Y(s)
$$

and, hence,

$$
U(s)=\left(s I-\Gamma D_{f}\right)^{-1} \Gamma r(s)+\left(s I-\Gamma D_{f}\right)^{-1} \Gamma Y(s) .
$$

As $Y(s)=G_{m}(s) U(s)$, it can be further deduced that

$$
\begin{aligned}
Y(s)= & G_{m}(s)\left(s I-\Gamma D_{f}\right)^{-1} \Gamma r(s) \\
& +G_{m}(s)\left(s I-\Gamma D_{f}\right)^{-1} \Gamma Y(s)
\end{aligned}
$$

which implies

$$
\begin{aligned}
& \left(I-G_{m}(s)\left(s I-\Gamma D_{f}\right)^{-1} \Gamma\right) Y(s) \\
& \quad=G_{m}(s)\left(s I-\Gamma D_{f}\right)^{-1} \Gamma r(s) .
\end{aligned}
$$

It can be inferred from (25) that the control strategy presented in Fig. 1 is equivalent to that presented in Fig. 4, with

$$
K(s)=\left(s I-\Gamma D_{f}\right)^{-1} \Gamma .
$$

Therefore, proving the stability of the control strategy presented in Fig. 1 is equivalent to proving the stability of the control strategy presented in Fig. 4.

Now consider the set

$\mathcal{C}_{1} \triangleq\left\{R(s) \in \mathcal{R} H_{\infty}^{n \times n}: \mathrm{j}\left[R(\mathrm{j} \omega)-R^{*}(\mathrm{j} \omega)\right]>0, \forall \omega \in(0, \infty)\right\}$

which is a subset of $\mathcal{C}$, described in (1). It can be shown that, if $P(s) \in \mathcal{C}$ and $Q(s) \in \mathcal{C}_{1}$, and $P(\infty) Q(\infty)=0$, then a positive feedback connection of $P(s)$ and $Q(s)$ is internally stable if and only if $\lambda_{\max }(P(0) Q(0))<1$, where $\lambda_{\max }(P(0) Q(0))$ denotes the maximum eigenvalue of the product $P(0) Q(0)$. Formally stated this result is as follows:

Theorem: Given $P(s) \in \mathcal{C}$ and $Q(s) \in \mathcal{C}_{1}$ such that $P(\infty) Q(\infty)=0$, then the positive feedback connection of $P(s)$ and $Q(s)$ is internally stable if and only if eigenvalues of the matrix $P(0) Q(0)$ are strictly less than 1 .

Proof: Refer to [25].

Thus for the system in Fig. 4 to be stable, it is sufficient for the controller $K(s)$ to satisfy (i) $K(s) \in \mathcal{C}_{1}$, (ii) $K(\infty)=0$, and (iii) the eigenvalues of $G(0) K(0)$ are strictly less than 1 . It follows from (26) that $K(\infty)=0$. For $K(s)$ to be in $\mathcal{C}_{1}$, it must be stable and $K(\mathrm{j} \omega)$ must satisfy

$$
\mathrm{j}\left[K(\mathrm{j} \omega)-K^{*}(\mathrm{j} \omega)\right]>0 .
$$

It can be shown that, for $\Gamma$ and $D$ both symmetric, with $\Gamma$ being positive definite and $D_{f}$ being negative definite (or $-D_{f}$ being 
positive definite), i.e., for

$$
\Gamma>0
$$

and

$$
-D_{f}>0
$$

$K(s)$ would belong to $\mathcal{C}_{1}$. A proof of this is given in the Appendix. Furthermore, note that $K(0)=-D_{f}^{-1}$. Therefore, the eigenvalue condition $\lambda_{\max }\left(G_{m}(0) K(0)\right)<1$ is equivalent to $G_{m}(0)\left(-D_{f}\right)^{-1}<I$, which in turn is equivalent to

$$
G_{m}(0)<-D_{f}
$$

Remark 1: If the controllers $K_{1}(s)=\left[s I-\Gamma_{1} D_{f_{1}}\right]^{-1} \Gamma_{1}$ and $K_{2}(s)=\left[s I-\Gamma_{2} D_{f_{2}}\right]^{-1} \Gamma_{2}$ satisfy (28) individually, then so does $K^{(2)}(s)=K_{1}(s)+K_{2}(s)$. In fact, for any finite sum $K^{(n)}(s)=\sum_{k=1}^{n} K_{k}(s)$, with each $K_{k}(s)=$ $\left[s I-\Gamma_{k} D_{f_{k}}\right]^{-1} \Gamma_{k}$ satisfying (28), $K^{(n)}(s)$ would also satisfy (28). For $K^{(n)}(s)$ to provide closed-loop stability, the required conditions are $\Gamma_{k}>0,-D_{k}>0$, for $k=1,2,3, \ldots, n$ and $-\sum_{k=1}^{n} D_{f_{k}}^{-1}>G_{m}(0)^{-1}$. These constraints can also be posed as LMIs affine in the variables $\Gamma_{k}$ and $D_{f}^{-1}$.

Remark 2: If $g_{0}=\sup _{m} G_{m}(0)$ is known, then choosing $-D_{f}>g_{0}$ I would guarantee stability over any finite number of out of bandwidth modes. In any physical system, power and bandwidth constraints ensure that it is not possible to excite all the infinite modes of the system. Hence, for $-D_{f}>g_{0} \mathrm{I}$ and $\Gamma>0$, the IRC will guarantee closed-loop stability for all practical purposes.

\section{EXPERIMENTAL VALIDATION}

To study the performance of multivariable IRC, a cantilever beam representing a physical resonant system is used as an experimental testbed. This cantilever beam is clamped at one end and free at the other end, and is susceptible to high amplitude oscillations when disturbed. Two pairs of piezoelectric patches are glued to the beam. One pair is positioned close to the clamped end and the other pair close to the free end of the beam. For each pair, one piezoelectric patch is used as an actuator (where input signals are applied) and the other patch acts as a sensor (where output signals are recorded). The sensor and the actuator patches of each pair are bonded in a back to back fashion such that they form a collocated sensor/actuator pair. Another solitary piezoelectric patch is attached to the center of the beam and is driven by a voltage source $w$. This voltage $w$ is used to represent the effect of disturbances on the beam. Figs. 5 and 6 contain schematics of the beam set up and an actual picture of the beam, respectively.

The experimental setup is a three-input three-output multivariable system (see Fig. 7). The input $v_{1}$ and the output $v_{p 1}$, denote the voltages applied and measured, respectively, at the first collocated sensor/actuator pair, while $v_{2}$ and $v_{p 2}$ denote the voltages applied and measured at the second collocated pair. The third input $w$ is the disturbance acting on the beam and the output $y_{\text {tip }}$ is the displacement of the tip of the beam measured by a noncontact Polytec laser vibrometer (PSV-300). The laser vibrometer has a bandwith of $20 \mathrm{kHz}$ and a resolution of

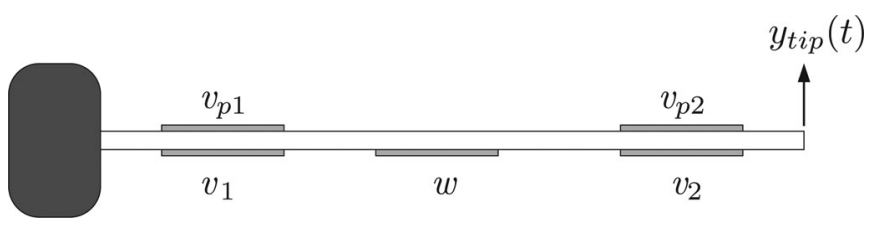

Fig. 5. Layout of the cantilever beam, $v_{1}$ and $v_{2}$ denote the actuator patches, $v_{p 1}$ and $v_{p 2}$ denote the sensor patches, $w$ denotes the noise patch and $y_{\text {tip }}(t)$ denotes tip displacement.

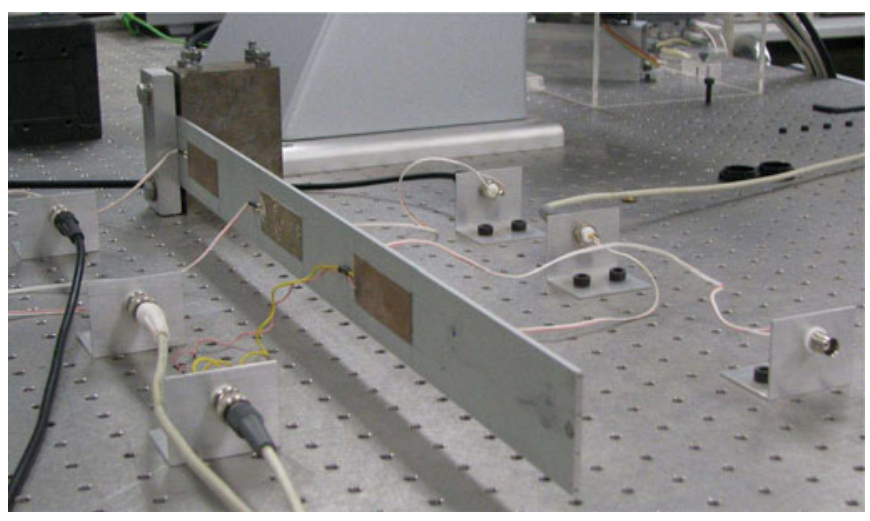

Fig. 6. Picture of the cantilever beam.

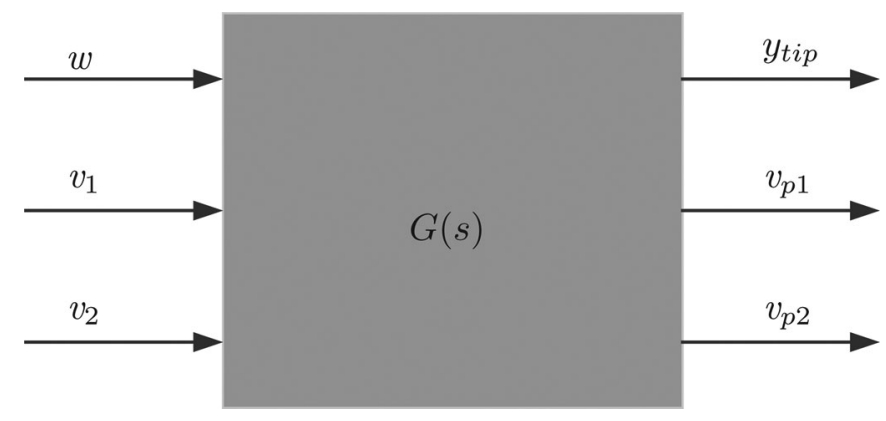

Fig. 7. Augmented MIMO plant.

TABLE I

BEAM PROPERTIES

\begin{tabular}{|l|c|}
\hline Length, $L$ & $550 \mathrm{~mm}$ \\
\hline Thickness, $h$ & $3 \mathrm{~mm}$ \\
\hline Width, $W$ & $50 \mathrm{~mm}$ \\
\hline Density, $\rho$ & $2.77 \times 10^{3} \mathrm{~kg} / \mathrm{m}^{3}$ \\
\hline Young's Mod., $E$ & $7.00 \times 10^{10} \mathrm{~N} / \mathrm{m}^{2}$ \\
\hline
\end{tabular}

$0.3 \mu \mathrm{m} / \mathrm{sec}$. The dimensions and properties of the the aluminum beam and the piezoelectric patches are tabulated in Tables I and II.

Even though this is a three-input three-output system, the noise input $w$ and the tip displacement are not directly used in the control design. Here, a positive feedback connection linking the output $V_{p}=\left[v_{p 1}, v_{p 2}\right]^{\top}$ to the input $u(t)=\left[v_{1}, v_{2}\right]^{\top}$, through a $2 \times 2$ controller $K(s)$, of the form described in (26), is designed to damp the resonant modes of the beam. The effectiveness of this control design is tested by exciting the beam through the noise patch and observing the corresponding tip response.

In Fig. 8, a schematic diagram of the experimental setup is presented. As shown there, the dSPACE board, which generates 
TABLE II

PIC 151 CERAMIC PROPERTIES

\begin{tabular}{|l|c|}
\hline Length, $L_{p z}$ & $50 \mathrm{~mm}$ \\
\hline Thickness, $h_{p z}$ & $0.25 \mathrm{~mm}$ \\
\hline Width, $W_{p z}$ & $25 \mathrm{~mm}$ \\
\hline Charge Constant, $d_{31}$ & $-210 \times 10^{-12} \mathrm{~m} / \mathrm{V}$ \\
\hline Voltage Constant, $g_{31}$ & $-11.5 \times 10^{-3} \mathrm{Vm} / \mathrm{N}$ \\
\hline Coupling Coefficient, $k_{31}$ & 0.34 \\
\hline Capacitance, $C_{p}^{S}$ & $115 \mathrm{nF}$ \\
\hline
\end{tabular}

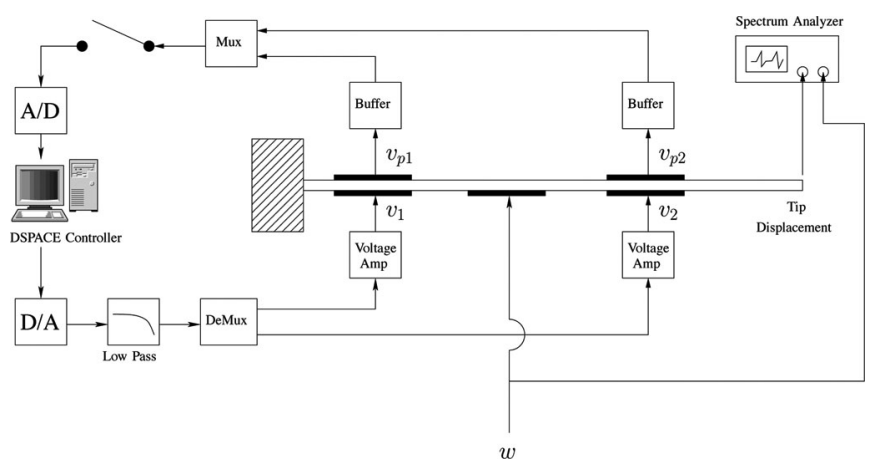

Fig. 8. Schematics of the experimental setup.

the control signal, is cascaded with a $20 \times$ voltage amplifier. dSPACE boards output a maximum of $\pm 10 \mathrm{~V}$ while the $20 \times$ voltage amplifier could output a maximum output of $\pm 200 \mathrm{~V}$. Thus, control signals with magnitude less than $200 \mathrm{~V}$ could be delivered without saturation.

\section{A. System Identification and Control Design}

Given that the structure is interpreted as a three-input threeoutput system, its associated frequency response function (FRF) $G(\mathrm{j} \omega)$ is a $3 \times 3$ matrix with each element $G_{i j}(\mathrm{j} \omega), i, j=1,2$, and 3 , corresponding to a particular combination of the input and the output, i.e.,

$$
G_{i j}(\mathrm{j} \omega)=G_{y_{i} u_{j}}=Y_{i}(\mathrm{j} \omega) / U_{j}(\mathrm{j} \omega)
$$

where $y_{1}=y_{\text {tip }}, y_{2}=v_{p 1}$, and $y_{3}=v_{p 2}$. Furthermore, $u_{1}=$ $w, u_{2}=v_{1}$, and $u_{3}=v_{2} . Y_{i}(\mathrm{j} \omega)$ and $U_{j}(\mathrm{j} \omega)$ are the Fourier transforms of $y_{i}$ and $u_{j}$, respectively. These FRFs are determined (nonparametrically) by applying swept sine waves, in the frequency band of $5-200 \mathrm{~Hz}$, to the piezoelectric actuators (including the central patch corresponding to the disturbance term $w)$ and measuring the corresponding output signals $y_{\text {tip }}, v_{p 1}$ and $v_{p 2}$. The inputs and the outputs, with the exception of $y_{\text {tip }}$, were generated and measured respectively using an HP36570A dual channel spectrum analyzer. In Fig. 9, the nonparametric FRFs $G_{i j}(\mathrm{j} \omega), i, j=1,2$ and 3 are plotted. It is apparent from the plots that all the FRFs have three resonance frequencies in the plotted frequency band, and not surprisingly the resonance frequencies are the same for all the FRFs.

Note that $\left\{v_{1}, v_{p 1}\right\}$ and $\left\{v_{2}, v_{p 2}\right\}$ are the only collocated pairs in the system. As mentioned above only the inputs $u(t)=\left[v_{1}, v_{2}\right]^{\top}$ and the outputs $V_{p}(t)=\left[v_{p 1}, v_{p 2}\right]^{\top}$ are used for control purposes. Therefore, to design a controller a model
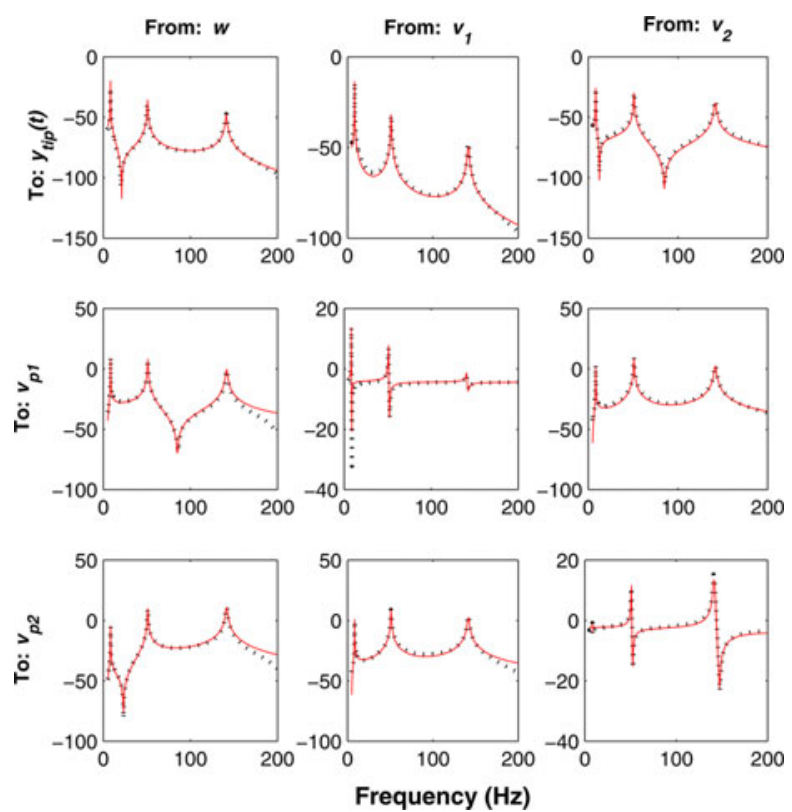

Fig. 9. Magnitude (in dB) plot of the identified model (solid) with the measured data (dotted).

of the form

$$
\begin{aligned}
\dot{x}(t) & =A x(t)+B u(t) \\
V_{p}(t) & =C x(t)+D u(t)
\end{aligned}
$$

is fitted to the frequency domain data $G_{i j}(\mathrm{j} \omega), i, j=2$ and 3 , plotted in Fig. 9. It can be observed from Fig. 9 that the model fits the data quite well. Since only three modes of the structure are modeled, $x$ is a $6 \times 1$ vector, $A$ is a $6 \times 6$ matrix, $B$ is $6 \times 2$ matrix, $C$ is $2 \times 6$ matrix, and $D$ is a $2 \times 2$ matrix. The model was fitted using the modified frequency domain subspace system identification algorithm presented in Section II. Matrices $A$ and $C$ were estimated using the first step of the standard frequency domain subspace identification method [32]. Matrix $D$ was obtained by numerically solving constrained convex optimization problem (7)-(9). The numerical solution was obtained using the Matlab package SeDuMi, [34].

Since $K(s)$ acts as a feedback controller connecting the plant output $V_{p}(t)$ to the input $u(t)$, it can be described in the state space form as

$$
\begin{aligned}
& \dot{\tilde{x}}=\Gamma D_{f} \tilde{x}+\Gamma V_{p} \\
& u=\tilde{x} .
\end{aligned}
$$

Setting $Z=[x, \tilde{x}]$ the closed-loop system can be written as

$$
\dot{Z}=\left[\begin{array}{cc}
A & B \\
\Gamma C & \Gamma\left(D+D_{f}\right)
\end{array}\right] Z .
$$

The goal is to choose $\Gamma$ and $D_{f}$ such that poles of (35) are well into to the left-half plane. To this end the following optimization problem is posed:

$$
\min _{\Gamma, D_{f}} \sum_{k=1}^{8}\left|P_{k}^{(d)}-P_{k}^{(c)}\left(\Gamma, D_{f}\right)\right|^{2}
$$




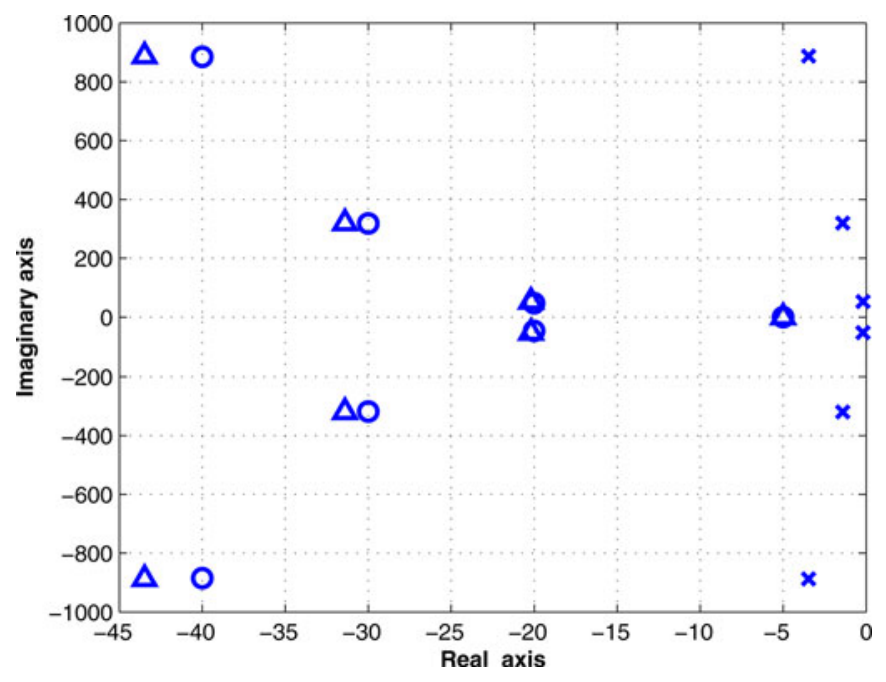

Fig. 10. Desired closed-loop poles $(\triangle)$ along with the open $(\times)$ and closedloop poles (०).

under the convex constraints, $\Gamma>0$ and $-D_{f}>G(0)=$ $-C A^{-1} B$. In (36), the $P_{k}^{(d)}$, s denote the set of desired closedloop poles, while $P_{k}^{(c)}\left(\Gamma, D_{f}\right)$ are the closed-loop pole positions for a given $\Gamma$ and $D_{f}$. In an ideal scenario one would like to have $\Gamma$ and $D_{f}$ such that $P_{k}^{(d)}=P_{k}^{(c)}\left(\Gamma, D_{f}\right)$ for all $k$. Here, the desired closed-loop poles are set to $\left\{P_{k}^{(d)}=p_{k}-\right.$ $20\}_{k=1}^{2}, \quad\left\{P_{k}^{(d)}=p_{k}-30\right\}_{k=3}^{4}$ and $\left\{P_{k}^{(d)}=p_{k}-40\right\}_{k=5}^{6}$, where $\left\{p_{k}\right\}_{k=1}^{2}$ are the open loop poles corresponding to the first resonant mode, $\left\{p_{k}\right\}_{k=3}^{4}$ are the open loop poles corresponding to the second resonant mode and $\left\{p_{k}\right\}_{k=5}^{6}$ are the open loop poles corresponding to the third resonant mode, and $P_{7}^{(d)}=P_{8}^{(d)}=-5$. In Fig. 10, the open- and the desired closedloop poles are plotted. The cost function is minimized using a nonlinear search with the constraints enforced. Needless to say, the cost function (36) is nonconvex and has many local minima. Therefore, the controller obtained here is only locally optimal.

It is worth noting that the closed-loop system (35), can also be written as

$$
\begin{aligned}
\dot{Z} & =\left(\left[\begin{array}{cc}
A & B \\
0 & 0
\end{array}\right]+\left[\begin{array}{l}
0 \\
I
\end{array}\right]\left[\begin{array}{ll}
\Gamma & \Gamma\left(D+D_{f}\right)
\end{array}\right]\left[\begin{array}{ll}
C & 0 \\
0 & 0
\end{array}\right]\right) Z \\
& \triangleq(\tilde{A}+\tilde{B} \tilde{K} \tilde{C}) Z
\end{aligned}
$$

Therefore, the optimization problem (36) along with its associated LMI constraints can also be interpreted as a generalized pole placement problem via static output feedback, which is difficult to solve, [36]-[39].

A Bode plot of the pole optimized controller $K_{\text {IRC }}(s)$ obtained by minimizing (36) under the convex constraints, $\Gamma>0$ and $-D_{f}>G(0)$ is plotted in Fig. 11. The simulated closedloop poles, i.e., poles of the closed-loop system (35) with the optimized controller, are plotted in Fig. 10. It is apparent that this controller places the closed-loop poles close to the desired pole locations. In order to predict or simulate the damping introduced in the FRF $G_{y_{\text {tip }} w}(\mathrm{j} \omega) \triangleq G_{11}(\mathrm{j} \omega)$ relating $w$ and $y_{\text {tip }}(t)$
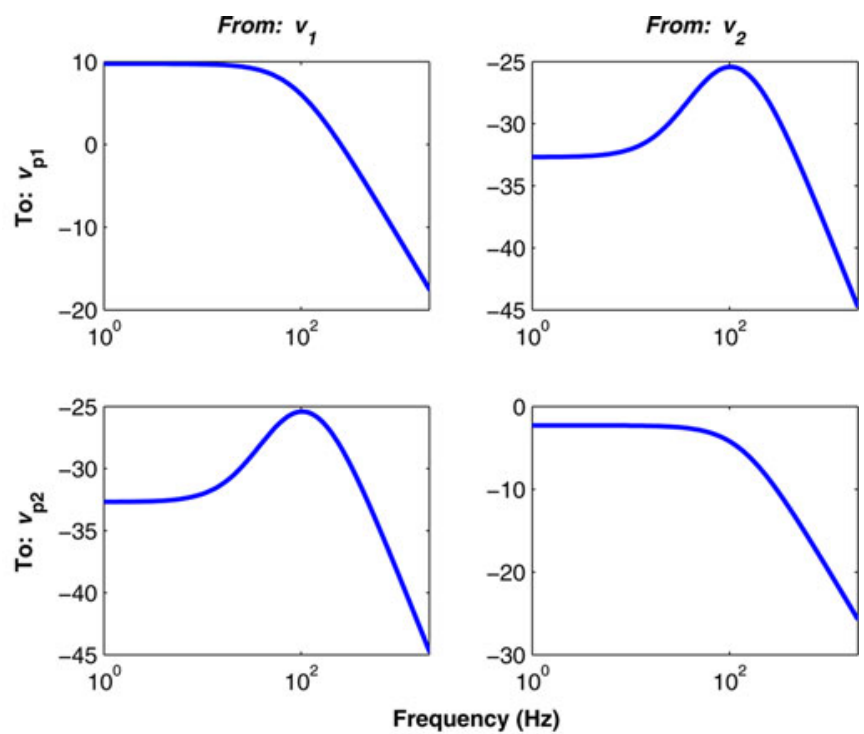

(a)
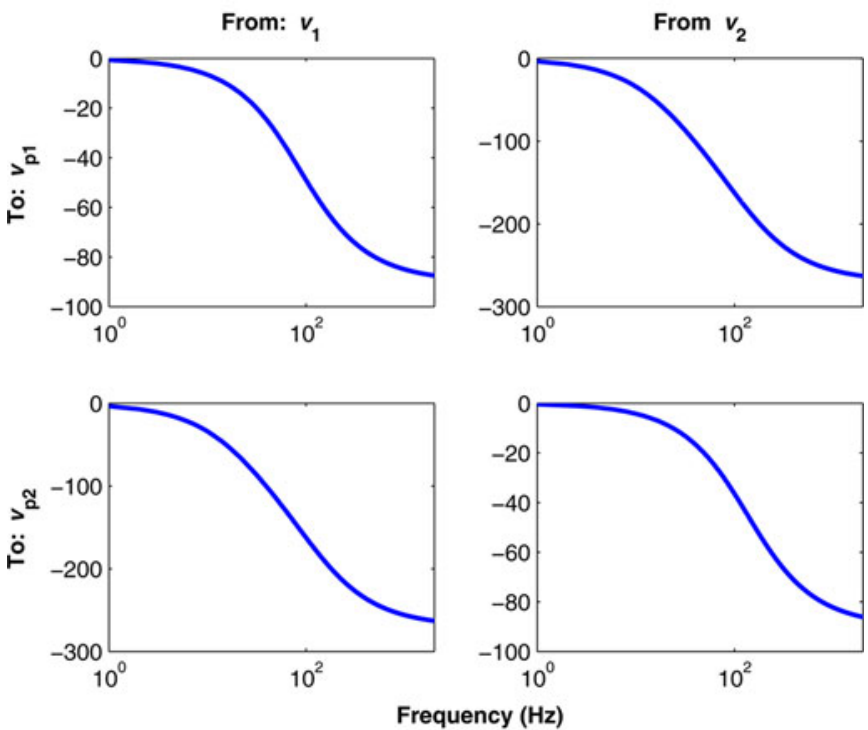

(b)

Fig. 11. (a) Magnitude (in dB) (b) Phase (in degs) plots of the pole optimized controller.

in closed loop, the $2 \times 2$ model (33) was extended to a $3 \times 3$ model by setting

$$
\begin{aligned}
\dot{x}(t) & =A x(t)+B_{w} w(t)+B u(t) \\
V_{p}(t) & =C x(t)+D_{v w} w(t)+D u(t) \\
y_{\text {tip }} & =C_{y} x(t)+D_{y w} w(t)+D_{y v} V(t)
\end{aligned}
$$

and estimating the unknown matrices $B_{w}, D_{v w}, C_{y}$, and $D_{y v}$ from the nonparametric data $G_{11}(\mathrm{j} \omega), G_{12}(\mathrm{j} \omega), G_{13}(\mathrm{j} \omega)$, $G_{21}(\mathrm{j} \omega)$, and $G_{31}(\mathrm{j} \omega)$. A Bode plot of this system is displayed in Fig. 9. Here, the estimation is done using standard nonlinear least squares. Using $K_{\mathrm{IRC}}(s)$, the pole optimized feedback controller, which is of the form (34), the complete $3 \times 3$ closed-loop 


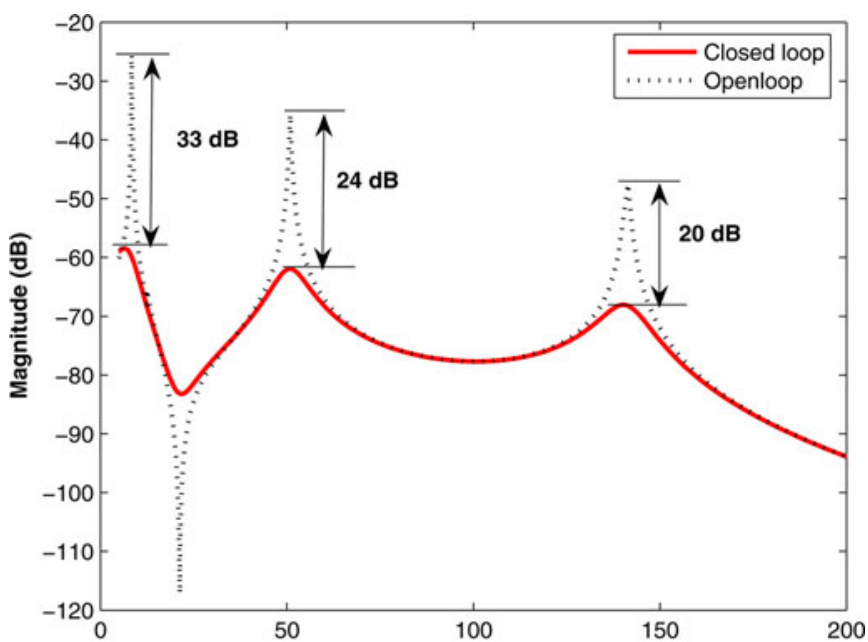

(a)

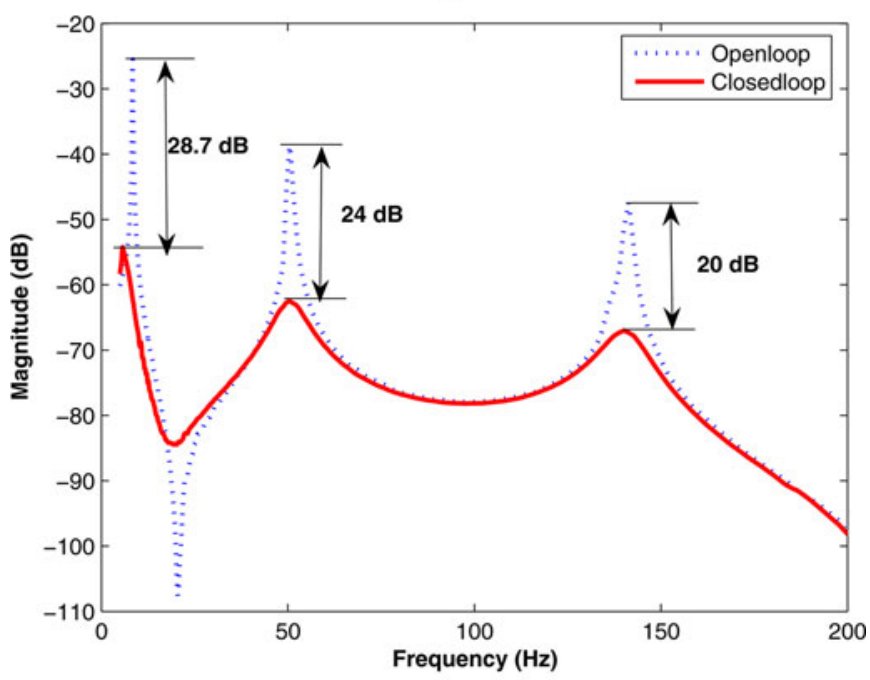

(b)

Fig. 12. (a) Simulated magnitude plot of the transfer function, $G_{y_{\mathrm{tip}} w}=$ $\left[y_{\text {tip }}(\mathrm{j} \omega) / w(\mathrm{j} \omega)\right]$ in open loop and in closed loop. (b) Experimentally determined magnitude plot of the transfer function, $G=\left[y_{\text {tip }}(\mathrm{j} \omega) / w(\mathrm{j} \omega)\right]$ in open loop and in closed loop.

system is written in the form

$$
\begin{aligned}
\dot{X}(t) & =\bar{A} X(t)+\bar{B}_{w} w(t) \\
y_{\mathrm{tip}}(t) & =\bar{C}_{y} X(t)+D_{y w} w(t)
\end{aligned}
$$

with $\bar{A}, \bar{B}, \bar{C}$, and $X(t)$ appropriately defined. In Fig. 12, the predicted closed-loop response $G_{y_{\mathrm{tip}} w}^{(\mathrm{Cl})}(\mathrm{j} \omega)$, which is the FRF corresponding to (39), is plotted along with the model fitted for $G_{y_{\text {tip }} w}(\mathrm{j} \omega)=G_{11}(\mathrm{j} \omega)$ plotted in Fig. 9. The closed-loop model predicts a damping of $33 \mathrm{~dB}, 24 \mathrm{~dB}$, and $20 \mathrm{~dB}$ in the first, second, and the third resonant modes, respectively.

The optimized controller is tested experimentally on the beam. In Fig. 12, plots of the FRF relating the $y_{\text {tip }}$ displacement and the noise input $w$ (see Fig. 7) both in open loop and in closed loop are presented. It can be observed that a substantial damping in all the three resonant modes is achieved. Also plotted in Fig. 12 is the simulated or predicted closed-loop re-

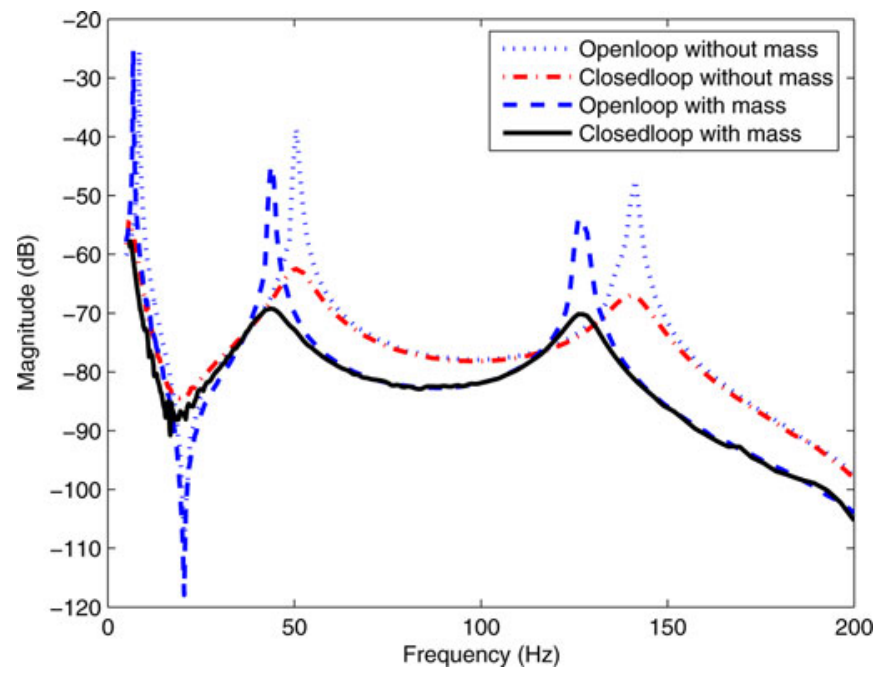

Fig. 13. Magnitude plot of the transfer function, $G=\left[y_{\operatorname{tip}}(\mathrm{j} \omega) / w(\mathrm{j} \omega)\right]$ in open loop and closed loop with and without mass.

sponse $G_{y_{\text {tip }} w}^{(C l)}(\mathrm{j} \omega)$, the FRF corresponding to (39). It can be observed that the predicted closed-loop response is reasonably close to the experimentally determined closed-loop response, except near the first resonance. This discrepancy is possibly due to modeling errors, and is not substantial.

A good controller must be robust to variations in plant dynamics. In practice, due to wear and tear and also due to variations in the environmental conditions, the dynamics of the plant tend to change. In particular, piezoelectric transducers are known to be rather sensitive to temperature variations [40]. These variations are known to result in a shift in resonance frequencies of the base structure [41], [42]. A good controller must be robust to these changes and provide sufficient damping even under these changed circumstances. In order to check the robustness properties of our controller, artificial changes in resonance frequencies were brought about by adding extra masses at the free end of the beam. In Fig. 13, the measured open-loop and closed-loop frequency response of the loaded system are presented. It must be stressed here that the controller used here is identical to that used for the unloaded cantilever beam in Fig. 12. The plots suggest that the IRC is robust to perturbations in resonance frequencies.

Finally, to illustrate the controller performance in the time domain, a pulse-shaped voltage signal was applied to the piezoelectric patch corresponding to the disturbance input $w$ of the beam. The resulting tip velocities, $\dot{y}_{\text {tip }}(t)$, in open and closed loop were recorded for the beam. These time-domain results are plotted in Fig. 14. They demonstrate the effectiveness of the controller in rejecting disturbances.

\section{CONCLUding Discussion}

In this paper, flexible structures with collocated sensor/actuator pairs were considered. Collocated sensor/actuator pairs lead to negative imaginary systems. Using the characterizations presented in [25] on negative imaginary systems, a novel identification scheme based on the frequency domain subspace method was presented. The novelty is in the convexification 


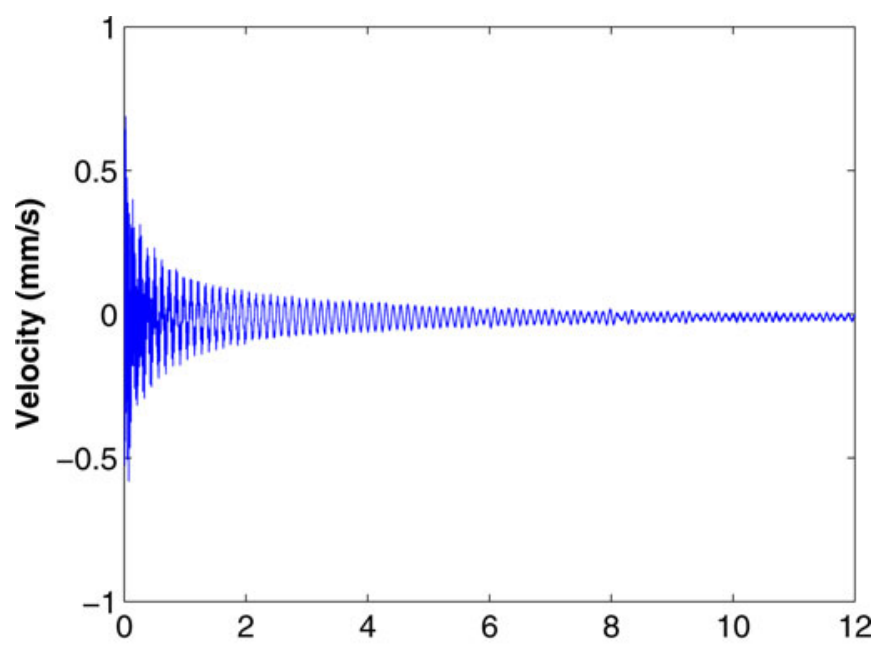

(a)

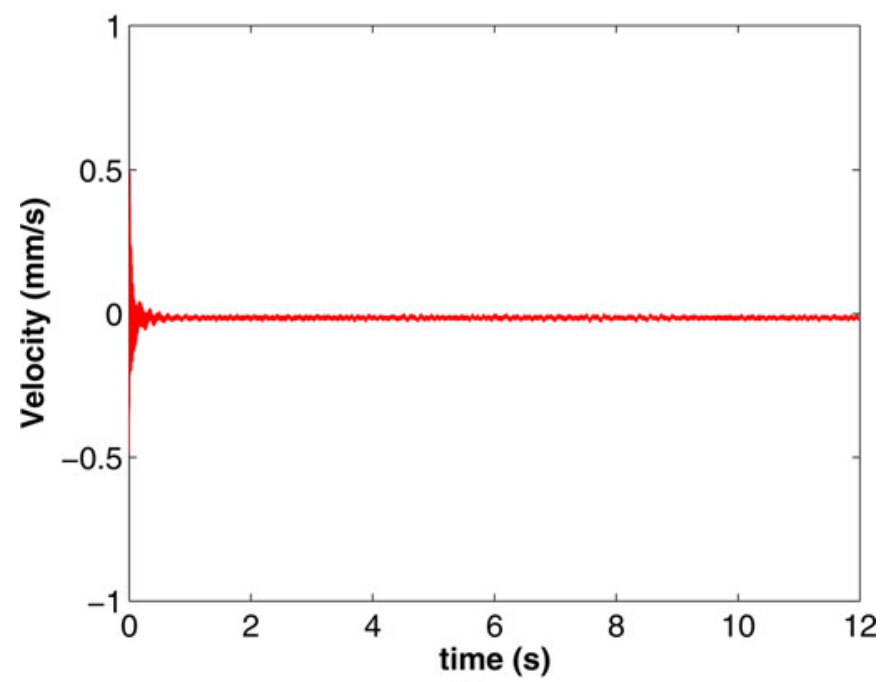

(b)

Fig. 14. Measured pulse response (a) in open loop, (b) in closed loop.

of the least squares estimation involved in the subspace method. The rudimentary IRC presented in [26] was reviewed and a formal mathematical framework for analyzing the control technique was presented. The IRC technique was generalized to MIMO systems with collocated sensors/actuators. The generalization from SISO systems (with one collocated sensoractuator pair) to the MIMO systems (with several collocated sensor-actuator pairs), though a natural progression, was a result of the mathematical framework provided for analyzing IRC. It was shown that for collocated resonant structures IRC controller guarantees closed-loop stability subject to certain LMI constraints, thereby making the class of such stabilizing controllers a convex set. A constrained optimization procedure was proposed for designing multivariable IRCs which can deliver sufficient damping. A multivariable IRC was designed for and implemented on an active structure with two pairs of collocated piezoelectric sensor/actuator pairs. The controller were shown to add significant damping to the active structure.
The procedure presented in this paper for designing IRCs involves minimizing a nonlinear nonconvex cost function over a convex set. The nonconvex nature of the cost function makes the control design reliant on the initial guess or the starting point of the nonlinear search used for minimizing the cost function. A convex optimization approach to IRC design is still an open question.

\section{APPENDIX}

In the following, it will be shown that

$$
K(s)=\left[s I-\Gamma D_{f}\right]^{-1} \Gamma
$$

belongs to the set

$$
\begin{aligned}
& \mathcal{C}_{1} \triangleq\left\{R(s) \in \mathcal{R} H_{\infty}^{n \times n}: \mathrm{j}\left[R(\mathrm{j} \omega)-R^{*}(\mathrm{j} \omega)\right]>0\right. \\
& \forall \omega \in(0, \infty)\}
\end{aligned}
$$

for

$$
\Gamma>0
$$

and

$$
-D_{f}>0 .
$$

In order to show that $K(s) \in \mathcal{C}_{2}$, it is enough to shown that $K(s)$ is stable and

$$
\mathrm{j}\left[K(\mathrm{j} \omega)-K^{*}(\mathrm{j} \omega)\right]>0 .
$$

Note that (42) and (43) imply that the product $-\Gamma D_{f}$ has strictly positive eigenvalues. Hence, stability of $K(s)$ follows directly from (40). As $\Gamma$ and $-D_{f}^{-1}$ are symmetric and positive definite, there exists a nonsingular $n \times n$ matrix $X$ such that

$$
X^{\top} D_{f}^{-1} X=\Lambda
$$

and

$$
X^{\top} \Gamma X=\mathrm{I}
$$

where $\Lambda$ is a diagonal matrix and $I$ is the identity matrix. The elements of $\Lambda$ are eigenvalues of $\Gamma^{-1} D_{f}^{-1}$ (see [43]). As $\Gamma$ and $-D_{f}$ are positive definite, every eigenvalue of the product $\Gamma D_{f}$, and hence $(\Gamma D)_{f}^{-1}$, is negative (see [44]). Therefore, $\Lambda=$ $\operatorname{diag}\left(-\lambda_{11},-\lambda_{22}, \ldots,-\lambda_{n n}\right)$, for some $\lambda_{k k}$ ' strictly positive.

Note that $\Gamma=X^{-\top} X^{-1}$ and $D_{f}^{-1}=X^{-\top} \Lambda X^{-1}$, which implies $D_{f}=X \Lambda^{-1} X^{\top}$ and $\Gamma D_{f}=X^{-\top} \Lambda^{-1} X^{\top}$. Hence,

$$
\begin{aligned}
K(s) & =\left[s I-\Gamma D_{f}\right]^{-1} \Gamma \\
& =\left[s I-X^{-\top} \Lambda^{-1} X^{\top}\right]^{-1} X^{-\top} X^{-1} \\
& =X^{-\top}\left[s I-\Lambda^{-1}\right]^{-1} X^{-1} .
\end{aligned}
$$

This, in turn, implies

$$
K(\mathrm{j} \omega)=\mathrm{X}^{-\top}\left[\mathrm{j} \omega \mathrm{I}-\Lambda^{-1}\right]^{-1} \mathrm{X}^{-1}
$$

and

$$
K^{*}(\mathrm{j} \omega)=\mathrm{X}^{-\top}\left[-\mathrm{j} \omega \mathrm{I}-\Lambda^{-1}\right]^{-1} \mathrm{X}^{-1} .
$$


Hence,

$$
\begin{aligned}
\mathrm{j}\left[K(\mathrm{j} \omega)-\mathrm{K}^{*}(\mathrm{j} \omega)\right]=\mathrm{jX}^{-\top}\left(\left[\mathrm{j} \omega \mathrm{I}-\Lambda^{-1}\right]^{-1}\right. \\
\\
\left.-\left[-\mathrm{j} \omega \mathrm{I}-\Lambda^{-1}\right]^{-1}\right) \mathrm{X}^{-1} .
\end{aligned}
$$

Setting $\alpha_{k}=\frac{1}{\lambda_{k k}}, k=1,2, \ldots, n$, it can be seen that

$$
\begin{aligned}
& {\left[j \omega I-\Lambda^{-1}\right]^{-1}=\left[\begin{array}{cccc}
\frac{1}{j \omega+\alpha_{1}} & 0 & \cdots & 0 \\
0 & \frac{1}{j \omega+\alpha_{2}} & \cdots & 0 \\
0 & 0 & \ddots & 0 \\
0 & 0 & \cdots & \frac{1}{j \omega+\alpha_{n}}
\end{array}\right]} \\
& =\left[\begin{array}{cccc}
\frac{-\mathrm{j} \omega+\alpha_{1}}{\omega^{2}+\alpha_{1}^{2}} & 0 & \cdots & 0 \\
0 & \frac{-\mathrm{j} \omega+\alpha_{2}}{\omega^{2}+\alpha_{2}^{2}} & \cdots & 0 \\
0 & 0 & \ddots & 0 \\
0 & 0 & \ldots & \frac{-j \omega+\alpha_{n}}{\omega^{2}+\alpha_{n}^{2}}
\end{array}\right]
\end{aligned}
$$

It is evident from (50) and (51) that $\mathrm{j}\left[K(\mathrm{j} \omega)-\mathrm{K}^{*}(\mathrm{j} \omega)\right]$ is strictly positive definite. Thus, for $\Gamma$ and $-D_{f}$ positive definite $K(s) \in \mathcal{C}_{1}$.

\section{REFERENCES}

[1] L. Meirovitch, Elements of Vibration Analysis. Sydney, Australia: McGraw-Hill, 1986.

[2] S. O. R. Moheimani, D. Halim, and A. J. Fleming, Spatial Control of Vibration: Theory and Experiments. Singapore: World Scientific, 2003.

[3] A. Preumont, Vibration Control of Active Structures: An Introduction. Dordrect, The Netherlands: Kluwer, 1997.

[4] B. Bhushan, Ed., Springer Handbook of Nanotechnology. Heidelberg, Germany: Springer-Verlag, 2004

[5] S. Wu, T. L. Turner, and S. A. Rizzi, "Piezoelectric shunt vibration damping of an F-16 panel under high acoustic excitation," Smart Struct. Mater.: Damping Isolation, Proc SPIE, vol. 3989, pp. 276-287, 2000.

[6] C. W. deSilva, Vibration Fundamentals and Practice. Boca Raton, FL: CRC Press, 1999.

[7] F. Casella, A. Locatelli, and N. Schiavoni, "Modeling and control for vibration suppression in a large flexible structure with jet thrusters and piezoactuators," IEEE Trans. Control Syst. Technol., vol. 10, no. 4, pp. 589-599, Jul. 2002.

[8] G. M. Clayton and S. Devasia, "Conditions for image-based identification of spm-nanopositioner dynamics," IEEE Trans. Mechatronics, vol. 14, no. 4, pp. 405-413, Aug. 2009.

[9] Chibum Lee and S. M. Salapaka, "Fast robust nanopositioning linearmatrix-inequalities-based optimal control approach," IEEE Trans. Mechatronics, vol. 14, no. 4, pp. 414-422, Aug. 2009.

[10] J. Juang and M. Q. Phan, Identification and Control of Mechanical Systems, 2001.

[11] W. Gawronski, Balanced Control of Flexible Structures. Heidelberg, Germany: Springer-Verlag, 1996.

[12] M. I. Friswell, D. J. Inman, and R. W. Rietz, "Active damping of thermally induced vibrations," J. Intell. Mater. Syst. Struct., vol. 8, no. 8, pp. 678685, Aug. 1997.

[13] D. Halim and S. O. R. Moheimani, "Spatial resonant control of flexible structures-application to a piezoelectric laminate beam," IEEE Trans. Control Syst. Technol., vol. 9, no. 1, pp. 37-53, Jan. 2001.
[14] D. Halim and S. O. R. Moheimani, "Experimental implementation of spatial $H_{\infty}$ control on a piezoelectric laminate beam," IEEE/ASME Trans. Mechatronics, vol. 7, no. 3, pp. 346-356, Sep. 2002.

[15] S. K. Kwak, G. Washington, and R. K. Yedavalli, "Acceleration feedbackbased active and passive vibration control of landing gear components," J. Aerosp. Eng., vol. 15, no. 1, pp. 1-9, 2002.

[16] D. Halim and S. O. R. Moheimani, "Spatial $\mathrm{H}_{2}$ control of a piezoelectric laminate beam: Experimental implementation," IEEE Trans. Control Syst. Technol., vol. 10, no. 4, pp. 533-546, Jul. 2002.

[17] A. G. Kelkar and S. M. Joshi, "Control of elastic systems via passivitybased methods," J. Vibr. Control, vol. 10, no. 11, pp. 1699-1735, Nov. 2004

[18] P. C. Hughes, "Space structure vibration modes: How many exist? which ones are important?" IEEE Control Syst. Mag., vol. 8, no. 1, pp. 22-28, Feb. 1987.

[19] M. J. Balas, "Feedback control of flexible systems," IEEE Trans. Autom Control, vol. 23, no. 6, pp. 673-679, Aug. 1978.

[20] M. J. Balas, "Active control of flexible systems," J. Optim. Theory Appl., vol. 25, no. 3, pp. 413-436, 1978.

[21] S. O. R. Moheimani and B. J. G. Vautier, "Resonant control of structural vibration using charge-driven piezoelectric actuators," IEEE Trans. Control Syst. Technol., vol. 13, no. 6, pp. 1021-1035, Nov. 2005.

[22] J. L. Fanson and T. K. Caughey, "Positive position feedback control for large space structures," AIAA J., vol. 28, no. 4, pp. 717-724, 1990.

[23] S. O. R. Moheimani, B. J. G. Vautier, and B. Bhikkaji, "Experimental implementation of extended multivariable PPF control on an active structure," IEEE Trans. Control Syst. Technol., vol. 14, no. 3, pp. 443-445, May 2006.

[24] A. J. den Hamer, G. Z. Angelis, and N. B. Roozen, "Broad-Band active vibration supression using PPF focused on industrial application," IEEE/ASME Trans. Mechatronics, vol. 10, no. 2, pp. 146-152, Apr. 2005.

[25] A. Lanzon and I. R. Petersen, "Stability robustness of a feedback interconnection of systems with negative imaginary frequency response," IEEE Trans. Autom. Control, vol. 53, no. 4, pp. 1042-1046, May 2008.

[26] S. S. Aphale, A. J. Fleming, and S. O. R. Moheimani, "Integral resonant control of collocated smart structures," Smart Mater. Struct., vol. 16, pp. 439-446, 2007

[27] B. Bhikkaji and S. O. R. Moheimani, "Integral resonant control of a piezoelectric tube actuator for fast nanoscale positioning," IEEE/ASME Trans. Mechatronics, vol. 13, no. 5, pp. 530-537, Oct. 2008

[28] P. A. Ioannau, H. Xu, and B. Fidan, "Identification and High Bandwidth Control of Hard Disk Drive Servo Systems with Sampled Data Measurements," IEEE Trans. Control Syst. Technol., vol. 15, no. 7, pp. 1089-1095, Nov. 2007.

[29] T. Atsumi, T. Arisaka, T. Shimizu, and H. Masuda, "Head positioning control in hard disk drives," IEEE/ASME Trans. Mechatronics, vol. 10, no. 4, pp. 378-384, Aug. 2005

[30] A. J. Fleming, "Nanopositioning system with force feedback for highperformance tracking and vibration control," IEEE Trans. Mechatronics, vol. 15 , no. 3, pp. 433-447, Jun. 2010.

[31] T. McKelvey, H. Akcay, and L. Ljung, "Subspace based multivariable system identification from system response data," IEEE Trans. Autom. Control, vol. 41, no. 7, pp. 960-979, Jul. 1996.

[32] L. Ljung, System Identification: Theory for the User. Upper Saddle River, NJ: Prenctice-Hall, 1999.

[33] T. McKelvey and S. O. R. Moheimani, "Estimation of phase constrained mimo transfer-functions with application to flexible structures with mixed collocated and non-collocated actuators and sensors," in Proc. of 16th IFAC World Congr., Prague, Czech Republic, Jul. 2005, p. Mo-MO2$\mathrm{TO} / 2$.

[34] J. F. Strum, "Using sedumi 1.02, a matlab tool for optimization over symmetric cones," Optim. Methods Softw., pp. 625-633, 1999.

[35] S. P. Bhattacharyya, H. Chapellat, and L. H. Keel, Robust Control: The Parametric Approach. $\quad$ Englewood Cliffs, NJ: Prentice Hall, 1995.

[36] V. L. Syrmos, C. Abdallah, and P. Dorato, "Static output feedback: a survey," Automatica, vol. 33, pp. 125-137, 1994.

[37] S. O. R. Moheimani and I. R. Petersen, "Optimal guaranteed cost control of uncertain systems via static and dynamic output feedback," Automatica, vol. 32, no. 4, pp. 575-579, 1996.

[38] V. D. Blondel and J. N. Tsitsiklis, "A survey of computational complexity results in systems and control," Automatica, vol. 36, pp. 1249-1274, 2000

[39] K. Yang and R. Orsi, "Generalized pole placement via static output feedback: A methodology based on projections," Automatica, vol. 42, pp. 2143-2150, 2006. 
[40] T. Ikeda, Fundamentals of Piezoelectricity. London, U.K.: Oxford Univ. Press, 1996.

[41] S. O. R. Moheimani, "A survey of recent innovations in vibration damping and control using shunted piezoelectric transducers," IEEE Trans. Control Syst. Technol., vol. 11, no. 4, pp. 482-494, Jul. 2003.

[42] D. Niederberger, A. J. Fleming, S. O. R. Moheimani, and M. Morari, "Adaptive multimode resonant piezoelectric shunt damping," Smart Mater. Struct., vol. 18, no. 2, pp. 291-315, Oct. 2004.

[43] A. J. Laub, Matrix Analysis for Scientists and Engineers. Philadelphia, PA: SIAM, 2005

[44] D. S. Bernstein, Matrix Mathematics. Princeton, NJ: Princeton University Press, 2005.

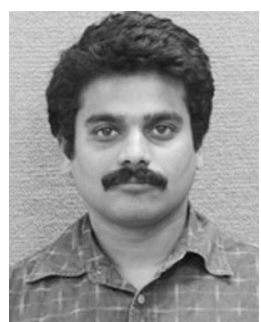

flexible structures.

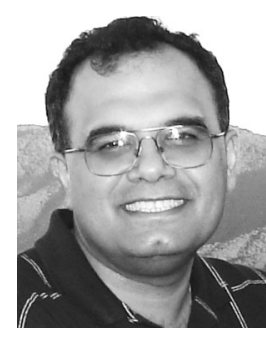

S. O. Reza Moheimani (F'11) received the Ph.D. degree in electrical engineering from the University of New South Wales at the Australian Defence Force Academy, Canberra, Australia in 1996

Since 1997, he has been with the University of Newcastle, Callaghan, Australia, where he is currently a Professor and Australian Research Council Future Fellow in the School of Electrical Engineering and Computer Science. His current research interests include applications of control and estimation in nanoscale positioning systems for scanning probe microscopy, control of microactuators in MEMS, and data storage systems. He has served on the Editorial Boards of a number of journals, including the IEEE TRANSACTIONS ON CONTROL SYSTEMS TECHNOLOGY, IEEE/ASME TRANSACTIONS ON MECHATRONICS, and Control Engineering Practice. He is a recipient of the 2007 IEEE TRANSACTIONS ON CONTROL SYSTEMS TECHNOLOGY Outstanding Paper Award and the 2009 IEEE Control Systems Technology Society Award.

Prof Moheimani is a Fellow of the Institute of Physics.

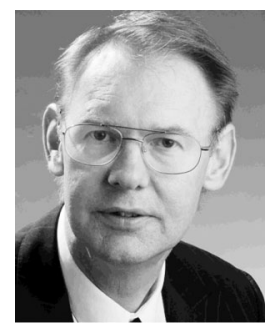

Ian R. Petersen (F'00) was born in Victoria, Australia. He received the Ph.D degree in electrical engineering from the University of Rochester, Rochester, NY, in 1984

From 1983 to 1985 , he was a Postdoctoral Fellow at the Australian National University. In 1985, he joined the University of New South Wales at the Australian Defence Force Academy, Canberra, Australia, where he is currently a Scientia Professor and an Australian Research Council Federation Fellow in the School of Information Technology and Electrical Engineering. He has served as an Associate Editor for the IEEE Transactions on Automatic Control, Systems and Control Letters, Automatica, and SIAM Journal on Control and Optimization. Currently he is an Editor for Automatica. His main research interests are in robust control theory, quantum control theory, and stochastic control theory. 